\title{
ULTRASONIC GUIDED WAVES FOR AGING WIRE INSULATION ASSESSMENT
}

\author{
Robert F. Anastasi ${ }^{l}$ and Eric I. Madaras ${ }^{2}$ \\ ${ }^{1}$ U.S. Army Research Laboratory, Vehicle Technology Directorate, AMSRL-VT-S, \\ Nondestructive Evaluation Sciences Branch, NASA Langley Research Center, Hampton, \\ VA 23681 \\ ${ }^{2}$ NASA Langley Research Center, Nondestructive Evaluation Sciences Branch, NASA, \\ Hampton, VA 23681
}

\begin{abstract}
Environmentally aged wire insulation can become brittle and crack and thus expose the underlying conductive wire to the potential for short circuits and fire. The feasibility of using ultrasonic guided waves to measure insulation condition was examined. First a simple model to study guided wave propagation in a bare and thin plastic coated wire was examined and then some aviation grade wire samples that had been heat-damaged. Initial measurements indicate that ultrasonic guided wave velocity can be used to monitor insulation stiffness.
\end{abstract}

\section{INTRODUCTION}

Electrical wiring is critical to the operation of most modern day equipment. Wiring is subjected to heat, cold, moisture, and vibrations, which can eventually cause the wire insulation and even the wire conductor to fail. In most cases these environmental and operational conditions are modest, but in some cases these conditions are extreme and can cause the insulation to become brittle and crack. The cracks expose the underlying wire conductor and become a potential source for short circuits and fire. Typical wire inspections are done visually and often after-the-fact, in response to an instrument or system failure. The visual inspection will find the cracks and burns, but offers little quantitative information about the condition of the wire insulation.

In its basic geometry the insulated wire may be considered a cylindrical wave-guide or more descriptively a clad rod, where the wire conductor is the core and the wire insulation is the cladding. A number of researchers have examined acoustic guided wave propagation in a cylindrical geometry [1-4] and for detailed analysis the reader is referred 
to these papers. Some applications of ultrasonic guided waves include material testing or characterization of wire $[5,6]$ or fibers, and for use as ultrasonic delay lines.

In general many acoustic wave modes will propagate in an isotropic cylinder and in part be a function of material property, geometry, frequency, propagation order, and circumferential order. Modes with circumferential order of zero are axial symmetric mode and can be divided into axial-radial and torsional. Modes of circumferential order one are flexural modes and modes of circumferential order two and higher are screw modes [2]. The lowest propagation order of the axial-radial mode extends to zero frequency where the limiting phase velocity is called the bar velocity. In the low frequency regime this mode is nearly non-dispersive. As frequency increases the phase velocity drops to a value slightly below the Rayleigh velocity and then approaches the Rayleigh velocity from below at higher frequencies.

In this paper the use of ultrasonic guided waves for detecting degradation in electrical wire insulation is examined. The concept is to generate an ultrasonic guided wave that will travel down a wire. Part of the wave will travel in the wire and part in the wire insulation. The condition of the wire insulation, its stiffness, will affect the overall wave speed and amplitude of the guided wave. Thus a measurement of wave speed will in part be a measurement of material stiffness or wire insulation condition.

\section{EXPERIMENTAL MEASUREMENTS AND RESULTS}

Initial measurements were carried out on a simple model of an insulated wire to identify various wave modes. This model consisted of a solid aluminum rod with a polymer coating. The aluminum rod, simulating the wire, had a $3.23 \mathrm{~mm}$ diameter. The polymer coating, simulating the wire insulation had a thickness of $0.57 \mathrm{~mm}$. The coating was a thermoplastic heat-shrink material of Polyolefin. Published [7] density and modulus of this material is $0.971 \mathrm{gm} / \mathrm{cm}^{3}$ and $1.2 \mathrm{Gpa}$ respectively, and the measured longitudinal wave velocity is $1870 \mathrm{~m} / \mathrm{s}$. The final diameter of the model was $4.37 \mathrm{~mm}$.

The experimental system is schematically shown in Figure 1. This system consists of two piezoelectric transducers, ultrasonic pulse generator, ultrasonic pre-amp, and oscilloscope. Transducers with a frequency range of $50 \mathrm{kHz}$ to $1.5 \mathrm{MHz}$ and were mechanically attached to the samples. A typical ultrasonic signal in the bare aluminum rod is shown in Figure 2. The smaller amplitude wave at about $50 \mu$ s is the first 


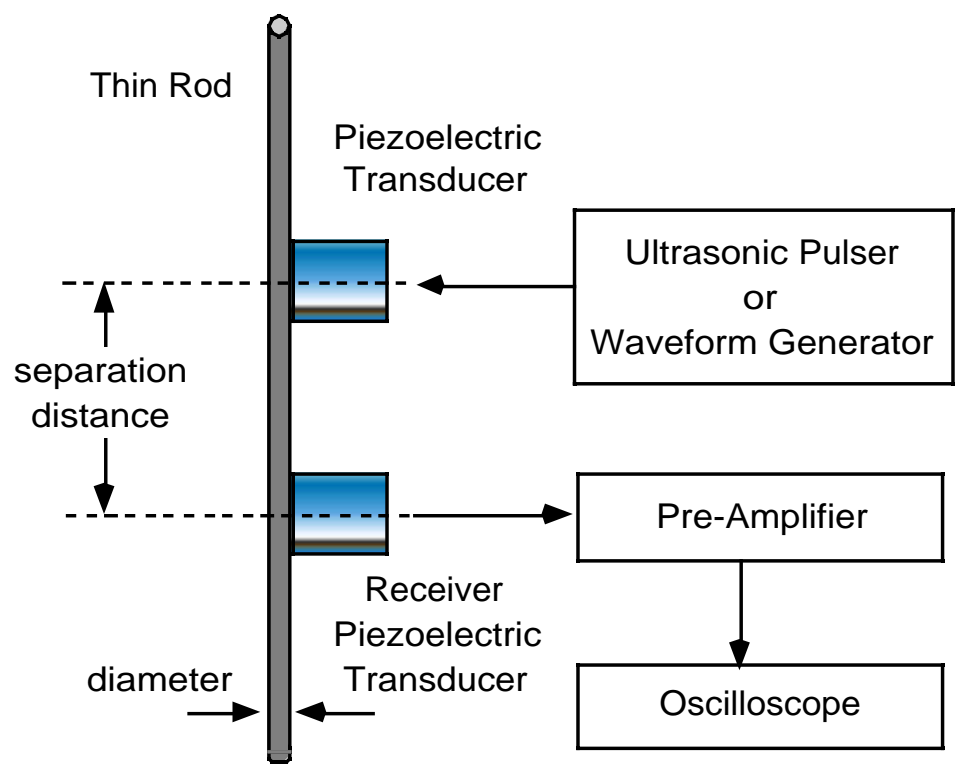

FIGURE 1. Schematic of experimental setup.

axisymmetric wave mode and the larger amplitude wave initiating at about $75 \mu$ s is the first flexural wave mode. The amplitude difference between the axisymmetric and flexural wave modes is consistent with the geometry of the ultrasonic generation. Since the transmitting receiver is located on the side of the rod, a larger amplitude bending force is applied to the rod and thus it was assumed a larger amplitude flexural mode would be generated. This assumption was checked by examining the signal as a function of rotational angle between the transmitting and receiving transducers. The transmitting transducer was held stationary while the receiving transducer rotated around the aluminum rod in increments of 10 degrees. A plot of the resulting axisymmetric and flexural mode amplitudes is shown in Figure 3. The axisymmetric mode amplitude is constant and the flexural mode amplitude follows a cosine-squared shape with a minimum at $90^{\circ}$. This is consistent with the assumption and the geometry of the loading.

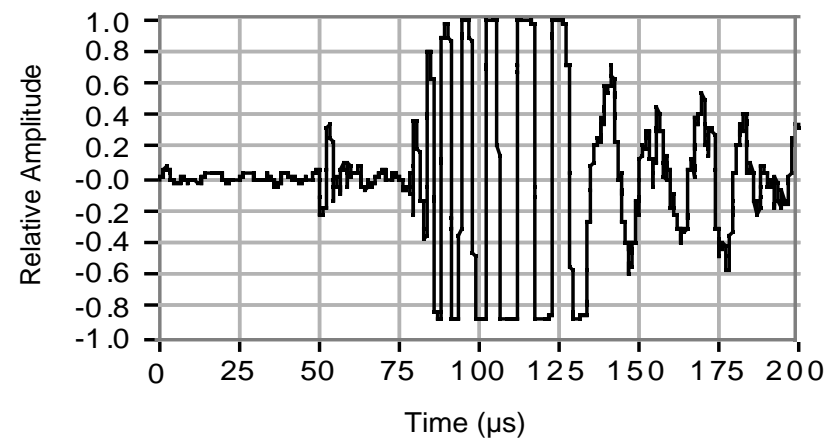

FIGURE 2. A typical ultrasonic signal in the bare aluminum rod showing the first axisymmetric wave mode and the first flexural mode wave. 


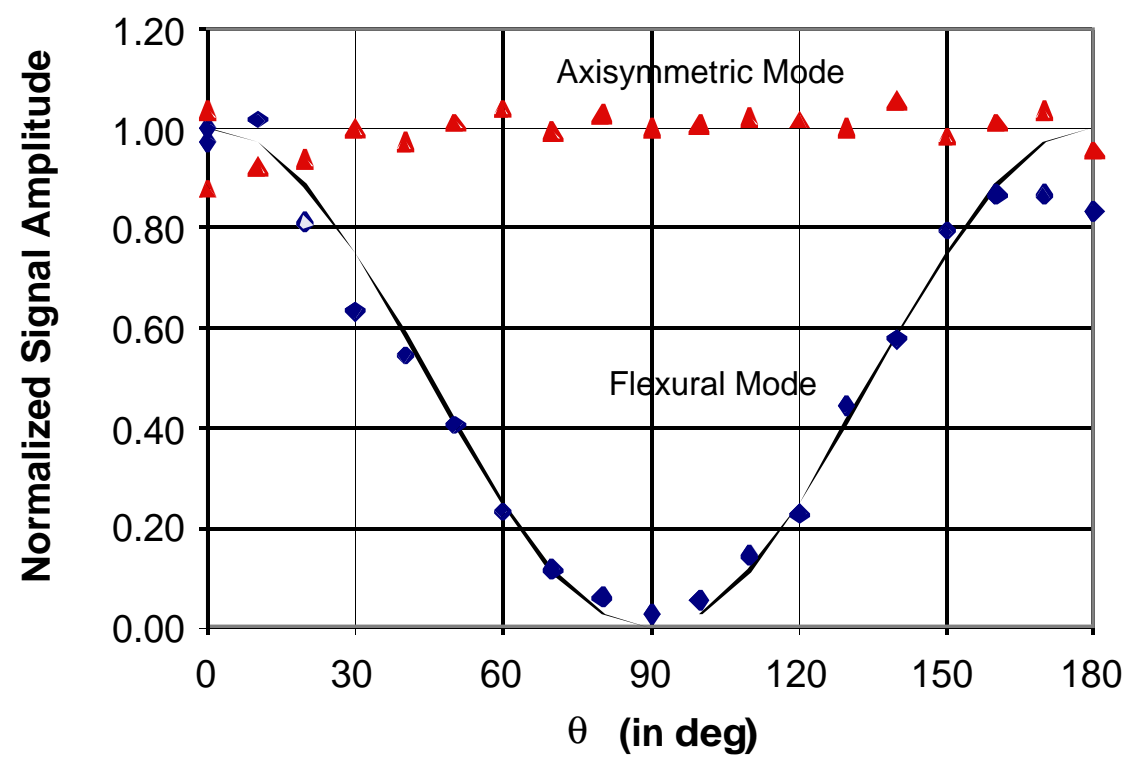

FIGURE 3. Plot showing the axisymmetric and flexural mode amplitudes as a function of angle between the transmitting and receiving transducers.

Signals similar to the one shown in Figure 2 were observed when the distance between the ultrasonic transmitter and receiver was varied. Analyzing these signals as a function of distance showed that the frequency content of the axisymmetric mode remained constant while the frequency content of the flexural mode varied and contained some higher order modes. These higher order modes were evident in the signal as small changes or variation in the sinusoidal shape of the wave and changed as the distance between the transducers varied.

The phase velocity of the axisymmetric mode was determined by taking a series of measurements of a constant phase point as a function of transducer separation. The separation distance varied from $50 \mathrm{~mm}$ to $250 \mathrm{~mm}$ over which 10 to 12 measurements were taken. The location of a constant phase point was plotted against the transducer separation and a linear curve fit was applied to the data. The slope of the linear fit was the measure of the phase velocity. The phase velocity of the bare rod and the polymer coated aluminum rod were $5128 \mathrm{~m} / \mathrm{s}$ and $4663 \mathrm{~m} / \mathrm{s}$, respectively. The bare aluminum rod phase velocity measurement is consistent with a calculated bar velocity of $5119 \mathrm{~m} / \mathrm{s}$. The aluminum properties used here were $70.76 \mathrm{Gpa}$ and $2.7 \mathrm{gm} / \mathrm{cm}^{3}$ for Young's Modulus and density, respectively. The measured changes in phase velocity between the bare and coated aluminum rod demonstrate the effect of the coating. It indicates that some of the ultrasonic energy is traveling in the insulation and maybe sensitive to stiffness changes in the wire insulation. 
To test this theory, some mil-spec wire samples were heat-damaged to change the condition in the insulation. It was assumed the heating did not change the insulation geometry or the boundary conditions between the insulation and the wire conductor because the temperature was not high enough to melt the insulation. The samples were 12,16 , and 20 gauge ethylene-tetraflouroethylene coated wire with the specification of MIL-W-22759/34. Table 1, shows the diameter, number of strands, and strand gauge as a function of wire gauge. Three samples of each gauge were cut to a length of approximately $60 \mathrm{~cm}$. One sample of each gauge was used for the baseline measurements, one of each gauge was heated in an oven at $349^{\circ} \mathrm{C}$ for one hour, and one of each gauge was heated in an oven at $399^{\circ} \mathrm{C}$ for one hour. The insulation on the baseline sample is smooth, flexible, and off-white in color. The samples heat-damaged at $349^{\circ} \mathrm{C}$ remained smooth and flexible, but its color changed to gray. The samples heatdamaged at $399^{\circ} \mathrm{C}$ became brittle, cracked, and its color became almost black. The phase velocity in these samples was measured following the same procedures described earlier and the results are shown in Figure 4. This figure is a bar chart showing phase velocity for each gauge family and each heat-damage condition. In each gauge family the baseline velocity is lowest, velocity increases for the $349^{\circ} \mathrm{C}$ heat-damage, and increases again for the $399^{\circ} \mathrm{C}$ heat-damage samples. This result shows that the axisymmetric phase velocity measurement is able to distinguish between the baseline and heat-damage conditions.

An alternate method of generating ultrasound is to use a low-power laser-diode. This method was previously demonstrated [8, 9] and was shown capable of generating ultrasound in aluminum and composite materials. The low-power and non-contact nature of this method makes it potentially easier to use than the clip-on transducers. As in the previous laser-diode ultrasound generation experiments a $150 \mathrm{~mW}$ modulated laser-diode to generate ultrasound and a conventional piezoelectric transducer as a receiver were used. A conventional ultrasonic signal was recovered by signal correlation.

TABLE 1. Insulated wire specifications.

\begin{tabular}{|l|r|r|r|}
\hline Gauge & $\mathbf{1 2}$ & $\mathbf{1 6}$ & $\mathbf{2 0}$ \\
\hline Overall Diameter, $(\mathrm{mm})$ & 2.78 & 1.90 & 1.51 \\
\hline Wire Bundle Dia., $(\mathrm{mm})$ & 2.05 & 1.29 & 0.81 \\
\hline Insulation Thickness, $(\mathrm{mm})$ & 0.365 & 0.305 & 0.35 \\
\hline Wire Strands per Bundle & 37 & 19 & 19 \\
\hline Wire Strand Gauge & 28 & 29 & 32 \\
\hline
\end{tabular}




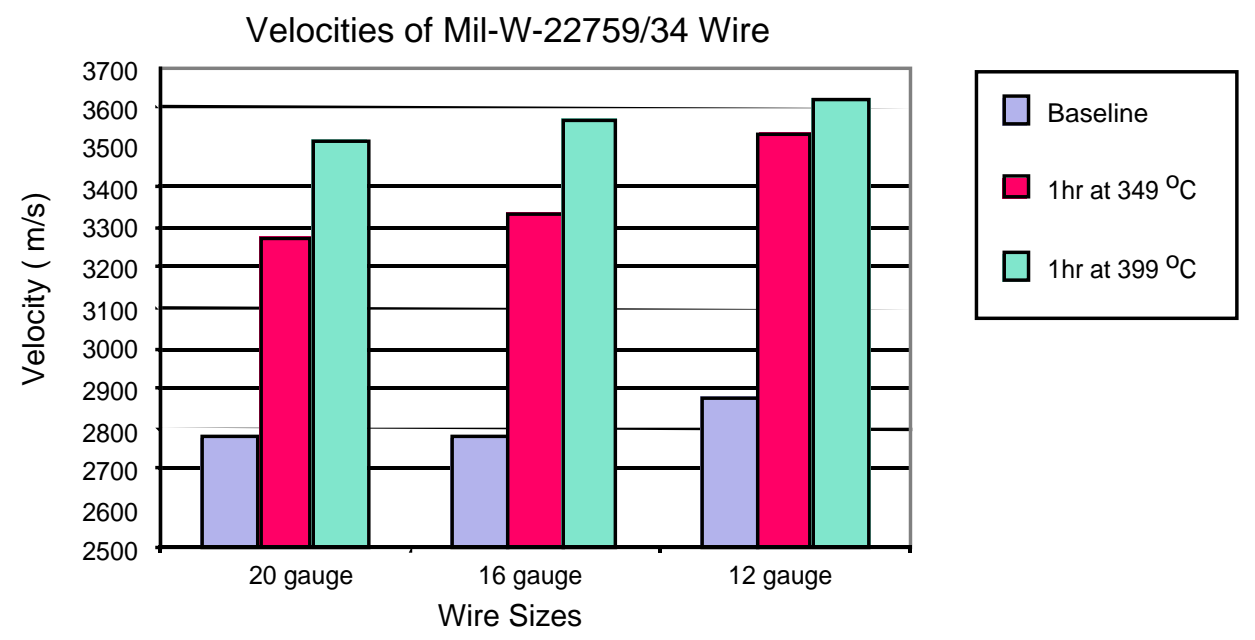

FIGURE 4. Bar chart showing phase velocity for each gauge family and each heat-damage condition.

The laser-diode beam incident on the wire insulation was $2 \mathrm{~mm}$ in diameter and had a power density of $17.83 \mathrm{~mW} / \mathrm{mm}^{2}$. A frequency generator to modulate the laser-diode drive current and thus the beam intensity was used in a frequency swept pattern from 1 $\mathrm{kHz}$ to $100 \mathrm{kHz}$. The insulation became damaged (slightly blackened) when the power density reached $20 \mathrm{~mW} / \mathrm{mm}^{2}$. The 12-gauge baseline sample and the 12-gauge sample that was heat-damaged at $349^{\circ} \mathrm{C}$ for one hour were examined. A typical ultrasonic signal recovered from correlating the drive and received signals is shown in Figure 5. The first flexural mode, the larger amplitude signal, can be seen initiating at about $120 \mu \mathrm{s}$. A fairly large amplitude signal can be seen at the beginning of the waveform and could be due in part to both signal processing and the electronics. The axisymmetric mode that was shown in previous measurements to be smaller in amplitude then the flexural mode could not be resolved. It is possible that the thermal generation mechanism lacked the energy to generate a symmetric wave.

The phase velocity of this flexural mode was measured by taking a series of measurements of a constant phase point as a function of generation point and receiver separation. The laser-diode was translated in millimeter increments and the piezoelectric ultrasonic receiver was held in a fixed position. The location of a constant phase point in time was plotted against the translation stage displacement and a linear curve fit was applied. The slope of the linear fit was our measure of the flexural phase velocity. The baseline flexural phase velocity was $529 \mathrm{~m} / \mathrm{s}$ while the heat-damaged sample had a phase velocity of $548 \mathrm{~m} / \mathrm{s}$. This measurement is consistent with previous measurements that showed an increase in phase velocity for the heat-damaged samples. 


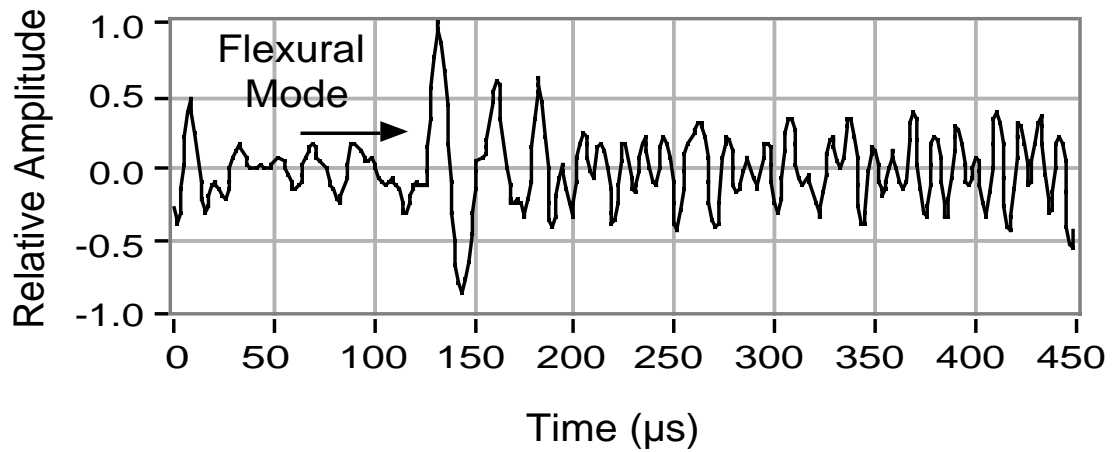

FIGURE 5. Laser-diode generated ultrasonic signal.

The flexural mode phase velocity measured with the laser is much slower than the axisymmetric mode phase velocity measured with the transducers. This is consistent with dispersion curve relations for cylindrical rods. These relations show that the flexural mode phase velocity approaches zero as frequency approaches zero while the axisymmetric mode phase approaches the bar velocity as the frequency approaches zero.

\section{CONCLUSION}

This work demonstrated the generation of ultrasonic axisymmetric and flexural guided wave in a plastic coated solid aluminum rod and in insulated wire samples. A clip-on piezoelectric transducer or a non-contact modulated low-power laser-diode was used for ultrasound generation. Axisymmetric and flexural guided wave modes were determined from the ultrasonic guided wave measurements on the bare aluminum rod. As the distance between the ultrasonic transmitter and receiver varied the axisymmetric wave mode shape and frequency content remained constant while the flexural guided wave mode dispersed thus its shape and frequency content varied. The phase velocity of both guided wave modes was effected by the addition of the plastic coating. In the heatdamaged wire experiments it was assumed the heating did not change the insulation geometry or the boundary conditions between the insulation and the wire conductor because the temperature was not high enough to melt the insulation. The axisymmetric phase velocity increased as the heat-damage increased. Thus measurements of the axisymmetric mode phase velocity may be sensitive to stiffness changes in the wire insulation. Similar changes in the flexural mode phase velocity were observed when the low-power laser-diode was used. The flexural mode was examined because the lasergenerated ultrasound method seemed to lack the energy to resolve the smaller amplitude axisymmetric waves. Since this flexural mode disperses, very small incremental measurement steps were required to follow the location of a constant phase point and 
determine the phase velocity. Frequency domain techniques could have been used to obtain the phase velocity more efficiently then the time domain method used. This would not change the results but potentially make them more accurate.

Although the heat-damage conditions are not the same as aging conditions with further development and refinements, the small clip on transducers and potentially the laserdiode-generated ultrasound can be used to inspect wire insulation for detrimental aging conditions.

\section{REFERENCES}

1. Meeker, T.R., and Meitzler, A.H., "Guided Wave Propagation in Eleongated Cylinders and Plates," in Physical Acoustics - Principles and Methods, edited by W.P. Nason, Academic Press, NY, Vol. 1, Part A, 1964, pp.111-167.

2. Thurston, R.N., J. Acoust. Soc. Am., 64, 1, 1-37, (1978).

3. McNiven, H.D., Sackman, J.L., and Shah, A.H., J. Acoust. Soc. Am., 35, 10, 16021609,(1963).

4. Abramson, H.N., J. Acoust. Soc. Am., 29, 1, 42-46, (1957).

5. Madaras, E. I., Kohl, T., and Rogers, W. P., "Material Property Characterization and Pulse Propagation in Thin Drawn Wire Waveguides," IEEE Ultrasonics Symposium1992, pp. 957-962.

6. Madaras, E. I., Kohl, T. W., Rogers, W. P., J. Acoust. Soc. Am., 97, 1, 252-261, (1995).

7. Electronic Source: The Online Materials Information Resource, http://www.matweb.com/SpecificMaterial.asp?bassnum=O4306\&group=General, Accessed July 5, 2001.

8. Madaras, E.I., and Anastasi, R.F., "Pseudo-Random Modulation of a Laser Diode for Generation Ultrasonic Longitudinal Waves," $26^{\text {th }}$ Annual Review of Progress in Qualitative Nondestructive Evaluation, Montreal, Quebec, Canada, July 1999.

9. Anastasi, R.F. and Madaras, E.I., "Pulse Compression Techniques for Laser Generated Ultrasound," IEEE International Ultrasonics Symposium-1999, edited by S.C. Schneider and B.R. McAvoy, IEEE Ultrasonics, Ferroelectronics, and Frequency Control Society, 1999. 